\section{Investigación Experimental o Metaanalítica}

Pensar en Movimiento:

Revista de Ciencias del Ejercicio y la Salud ISSN 1659-4436

Vol. 15, No.2, pp. 1-12

Abre el $1^{\circ}$ de julio, cierre al 31 de diciembre, 2017

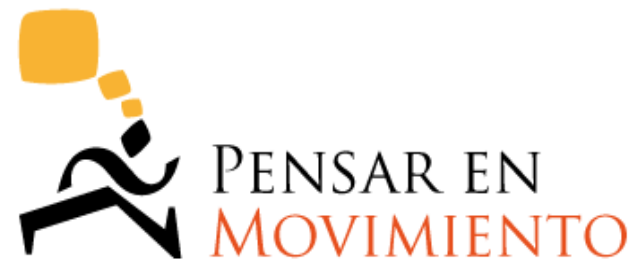

REVISTA DE CIENCIAS DEL EJERCICIO Y LA SALUD

\title{
EFECTO AGUDO DE DOS INTENSIDADES DE EJERCICIO CONTRA RESISTENCIA SOBRE LA PRESIÓN ARTERIAL EN REPOSO DE PERSONAS NORMOTENSAS
}

\section{ACUTE EFFECT OF TWO RESISTANCE EXERCISE INTENSITIES ON RESTING BLOOD PRESSURE IN NORMOTENSIVE INDIVIDUALS}

\section{EFEITO AGUDO DE DUAS INTENSIDADES DE EXERCÍCIO CONTRA RESISTÊNCIA SOBRE A PRESSÃO ARTERIAL EM REPOUSO DE PESSOAS NORMOTENSAS}

\author{
Mariana Gamboa-Granados, Bach. ${ }^{1(B, C, D, E)}$ y Andrea Solera-Herrera, Ph.D. ${ }^{1(A, B, D, E)}$ \\ andrea.solera@ucr.ac.cr \\ ${ }^{1}$ Centro de Investigación en Ciencias del Movimiento Humano, Universidad \\ de Costa Rica, San José, Costa Rica \\ Envío original: 11/07/2017; reenviado: 29/09/2017; \\ aceptado: 30/09/2017; publicado: 12/12/2017.
}

Doi: 10.15517/pensarmov.v15i2.29809

\section{RESUMEN}

Gamboa-Granados, M. y Solera-Herrera, A. (2017). Efecto agudo de dos intensidades de ejercicio contra resistencia sobre la presión arterial en reposo de personas normotensas. Pensar en Movimiento: Revista de Ciencias del Ejercicio y la Salud, 15(2), 1-12. El propósito del estudio fue determinar el efecto sobre la presión arterial (PA) del ejercicio contra resistencia $(\mathrm{CR})$ realizado con diferentes intensidades, en 10 hombres (38.22 \pm 7.03 años; $75.15 \pm 5.41 \mathrm{~kg} ; 1.69 \pm 0.07 \mathrm{~m}$; PA en reposo $116 \pm 7.5 / 71 \pm 7.3 \mathrm{mmHg}$ ), quienes participaron en cuatro sesiones de entrenamiento $\mathrm{CR}$ en días diferentes. La primera de ellas consistió en evaluar una repetición máxima (1RM) de cada uno de los ejercicios, mientras que las otras tres fueron realizadas en orden aleatorio y consistieron en: (a) ejecutar siete ejercicios CR (2 series $\times 14$ repeticiones) al $50 \%$ de $1 \mathrm{RM}$; (b) realizar los mismos siete ejercicios ( 2 series $\times 10$ 
repeticiones) al 70\% de $1 \mathrm{RM}$; y (c) permanecer en reposo durante $35 \mathrm{~min}$. Se tomó la PA en reposo antes del ejercicio, inmediatamente finalizado el ejercicio y, posterior al ejercicio, cada $10 \mathrm{~min}$, hasta completar $60 \mathrm{~min}$. Los ANOVAS de dos vías con medidas repetidas en ambos factores $(p<0.05)$ demostraron que: (1) la PA sistólica es significativamente menor a partir de los 20 min luego del ejercicio, cuando la persona se ejercita al $70 \%$, en comparación con la condición control y con la medición antes del ejercicio; (2) la PA diastólica es significativamente menor luego de realizar ejercicio, a ambas intensidades, con respecto a la medición antes del ejercicio y en comparación con la condición control (no obstante, los efectos son más duraderos luego de ejercitarse al 50\%). En conclusión, ambas intensidades de ejercicio desencadenan el efecto hipotensor postejercicio en varones normotensos.

Palabras claves: hipotensión postejercicio; presión arterial; ejercicio contra resistencia.

\section{ABSTRACT}

Gamboa-Granados, M. y Solera-Herrera, A. (2017). Acute Effect of Two Resistance Exercise Intensities on Resting Blood Pressure in Normotensive Individuals. PENSAR EN MovimiEnto: Revista de Ciencias del Ejercicio y la Salud, 15(2), 1-12. The aim of the study was to determine the effect of resistance-training exercise performed at different intensities on blood pressure (BP) in normotensives. Ten males (age $=38.2 \pm 7.0 \mathrm{yrs}$; body weight $=75.2 \pm 5.4 \mathrm{~kg}$; body height $=169 \pm 7.0 \mathrm{~cm}$; resting $\mathrm{BP}=116 \pm 7.5 / 71 \pm 7.3$ $\mathrm{mmHg}$ ) participated in four resistance-training exercise sessions performed in different days. The first session was used to determine one repetition maximum (1RM) for each resistance-training exercise; the following three sessions were randomly assigned and consisted of: a) seven resistance-training exercises performed at $50 \%$ of $1 \mathrm{RM}$ ( 2 sets $\mathrm{x}$ 14 reps); b) seven resistance-training exercises performed at $70 \%$ of $1 \mathrm{RM}$ ( 2 sets $\times 10$ reps); and c) control condition (35-min quiet resting). Resting BP was measured before exercise, immediately following exercise, and at 10-min intervals for 60-min. Two-way repeated measures ANOVAs showed $(p<0.05)$ that: $(1)$ the systolic BP significantly decreased 20-min following exercise in the 70\% 1RM condition compared to the control condition and the measurement before exercise; (2) the diastolic BP was significantly reduced following both exercise intensities compared to the measurements obtained before exercise and compared to the control condition. The acute effects lasted longer following exercise at $50 \%$ of $1 \mathrm{RM}$. In conclusion, both resistance-training exercise intensities elicited a post-exercise hypotensive effect in normotensive participants.

Key Words: post-exercise hypotension; blood pressure; resistance exercise.

\section{RESUMO}

Gamboa-Granados, M. y Solera-Herrera, A. (2017). Efeito agudo de duas intensidades de exercício contra resistência sobre a pressão arterial em repouso de pessoas normotensas. Pensar en Movimiento: Revista de Ciencias del Ejercicio y la Salud, 15(2), 1-12. O propósito do estudo foi determinar o efeito sobre a pressão arterial (PA) do exercício contra resistência $(C R)$ realizado com diferentes intensidades, em 10 homens $(38,22 \pm 7,03$ anos; $75,15 \pm 5,41 \mathrm{~kg} ; 1,69 \pm 0,07 \mathrm{~m}$; PA em repouso $116 \pm 7,5 / 71 \pm 7,3$ 
$\mathrm{mmHg}$ ), que participaram em quatro sessões de treinamento $\mathrm{CR}$ em dias diferentes. A primeira delas consistiu em avaliar uma repetição máxima (1RM) de cada um dos exercícios, enquanto outras três foram realizadas aleatoriamente e consistiram em: (a) executar sete exercícios CR (2 séries x 14 repetições) a 50\% de 1RM; (b) realizar os mesmos sete exercícios (2 series $\times 10$ repetições) a 70\% de 1RM; e (c) permanecer em repouso durante $35 \mathrm{~min}$. Mediu-se a PA em repouso antes do exercício, imediatamente depois de finalizá-lo e, posterior a seu término, a cada $10 \mathrm{~min}$, até completar $60 \mathrm{~min}$. Os ANOVAS de duas vias com medidas repetidas em ambos os fatores $(p<0,05)$ demostraram que: (1) a PA sistólica é significativamente menor a partir dos 20 min depois do exercício, quando a pessoa se exercita a 70\%, em comparação à condição controle e com a medição antes do exercício; (2) a PA diastólica é significativamente menor depois de realizar exercício, em ambas as intensidades, com relação à medição antes do exercício e em comparação à condição controle (entretanto, os efeitos são mais duradouro depois de exercitar-se a $50 \%$ ). Em conclusão, ambas as intensidades de exercício desencadeiam o efeito hipotensor pós-exercício em homens normotensos.

Palavras-chaves: hipotensão pós-exercício; pressão arterial; exercício contra resistência.

La hipertensión arterial es la enfermedad crónica con mayor prevalencia en el mundo (Fiedler y Gaurzong, 2005; Fuchs, 2005) y la exposición continua a ella aumenta el riesgo de padecer enfermedades renales, cardiovasculares y cerebrovasculares (James et al., 2014), por lo que se hace vital prevenir y retardar su aparición adoptando estilos de vida saludables que enfaticen en el aumento de la actividad física y la práctica regular de ejercicio (Chobanian et al., 2003; del Valle Soto, et al. 2015). Aunque muchos estudios abordan el efecto del ejercicio sobre la presión arterial (PA) de manera crónica -es decir, luego de varias semanas de realizarlo-, existen otros que lo miden en las horas siguientes a su ejecución (Cardoso et al., 2010).

La disminución sostenida de la PA en las horas posteriores al ejercicio recibe el nombre de "hipotensión post-ejercicio (HPE)" (Kenney \& Seals, 1993; Hamer, 2006) y recientemente está siendo estudiada, pues la evidencia científica sugiere que la magnitud del efecto agudo hipotensor podría predecir la magnitud de la disminución de la PA luego de realizar entrenamiento crónico (Liu, Goodman, Nolan, Lacombe \& Thomas, 2012), y los mecanismos fisiológicos que explican la reducción aguda y crónica de la PA también podrían estar relacionados (Hamer, 2006). Metodológicamente, la hipotensión postejercicio se determina comparando los niveles de presión arterial al terminar el ejercicio con los valores medidos antes de haber iniciado el ejercicio, o bien, contrastando los niveles de presión arterial del día en que la persona se ejercitó con los mostrados otro día en que no lo hiciera (Cardoso et al., 2010).

Aunque existe evidencia científica respaldando la HPE, los estudios actualmente se enfocan en determinar cuáles son las características que debe tener el ejercicio para lograr una mayor magnitud y duración de la HPE (Carpio, Moncada, Salazar, \& Solera, 2016), tanto en normotensos como en hipertensos, ya que se quiere ofrecer no solo el ejercicio como factor de protección para evitar el desarrollo de la enfermedad, sino 
también como terapia no farmacológica para quienes ya la han desarrollado (Del Valle, et al. 2015).

Mientras algunas investigaciones comparan con qué tipo de ejercicio se encuentra una mayor HPE, si con ejercicio aeróbico o contra resistencia (CR) (Bermudes, Vasallo, Vasquez, \& Lima, 2003; Keese, Farinatti, Pescatello, \& Monteiro, 2011), otros estudios van más allá y exploran si la intensidad a la que se realiza el ejercicio es un factor importante para desencadenar este fenómeno (Gamboa y Solera, 2014; Rezk, Marrache, Tinucci, Mion, \& Forjaz, 2006).

Autores como Keese et al. (2011) determinaron que la presión arterial sistólica (PAs) disminuye por un periodo de tiempo mayor (120 minutos) cuando se hace ejercicio aeróbico o concurrente, en comparación con cuando se hace ejercicio CR (80 min); mientras que la presión arterial diastólica (PAd) disminuye en 50 min, 40 min y 20 min, luego de realizar ejercicio aeróbico, concurrente o $\mathrm{CR}$, respectivamente. No obstante, las intensidades de trabajo de cada tipo de ejercicio fueron diferentes, por lo que no se podría atribuir con claridad si la diferencia de los efectos se debe al tipo de ejercicio o a la intensidad con que fueron realizados.

Una investigación realizada con 17 hombres normotensos, con una edad promedio de 23 años, encontró una disminución similar de la presión arterial sistólica si se realiza ejercicio $\mathrm{CR}$ al $40 \%$ y al $80 \%$ de $1 \mathrm{RM}$, mientras que la presión arterial diastólica disminuyó posterior al ejercicio de $40 \%$ de intensidad y se mantuvo estable luego del ejercicio al 80\% (Rezk, et al., 2006). En un estudio reciente se comparó el efecto de realizar ejercicio aeróbico a diferentes intensidades sobre la presión arterial en reposo de 10 hombres normotensos, cuya edad promedio era de $49.22 \pm 7.03$ años (Gamboa \& Solera, 2014), y se encontró que la presión arterial sistólica disminuyó significativamente durante $30 \mathrm{~min}$ y $60 \mathrm{~min}$ luego de realizar ejercicio al $50 \%$ y al $70 \%$ de la frecuencia cardiaca máxima, respectivamente; mientras que la presión arterial diastólica no presentó ningún cambio significativo.

Dado que diversos autores señalan la carencia de investigaciones sobre el efecto hipotensor del ejercicio posterior a una sesión de ejercicio CR (Del Valle et al., 2015), se diseñó este estudio, el cual pretende determinar el efecto de realizar ejercicio CR al $50 \%$ y $70 \%$ de 1 RM sobre la presión arterial en reposo de personas normotensas, intensidades sugeridas por diferentes organizaciones (por ejemplo, la Sociedad Española de Medicina del Deporte) como tratamiento en la prevención y tratamiento no farmacológico de la hipertensión.

\section{METODOLOGÍA}

Participantes. En el estudio participaron 10 hombres sanos, físicamente activos, que realizaban ejercicio al menos tres veces por semana, durante una hora, con experiencia en entrenamiento $\mathrm{CR}$, una edad promedio de $38.22 \pm 7.03$ años, un peso de $75.15 \pm 5.41 \mathrm{~kg}$, una estatura de $1.69 \pm 0.07 \mathrm{~m}$, un porcentaje de grasa de $28.62 \pm$ $4.28 \%$ y una PA en reposo de $116 \pm 7.5 / 71 \pm 7.3 \mathrm{mmHg}$.

Procedimientos e instrumentos de medición. El estudio contó con la aprobación del Comité Ético Científico de la Universidad de Costa Rica, de manera que en la primera sesión se les explicó a los participantes en qué consistía el estudio y se 
les invitó a firmar la hoja de consentimiento informado. Asimismo, se les solicitó a los voluntarios que se abstuvieran de realizar ejercicio o ingerir cafeína en las 24 horas previas a cada una de las condiciones y que no ingirieran ningún líquido o alimento durante las sesiones de ejercicio. Cada sujeto participó en cuatro sesiones, realizadas en días diferentes, con al menos tres días de separación entre sesión y sesión. Durante la primera sesión, al participante se le midió:

- La PA, con un medidor automático Marca OMRON modelo Bp791it.

- El peso y el porcentaje de grasa, con una báscula OMRON Body Composition MONITOR with Scale HBF-500INT.

- La fuerza máxima por medio de 1 RM para cada ejercicio que iba a realizar durante las sesiones experimentales. El protocolo de medición de 1RM consistió en un calentamiento de 10 min en banda sin fin, a una intensidad baja, y un calentamiento específico de 15 repeticiones con un peso bajo, previo a realizar la evaluación en cada ejercicio. Luego se le solicitaba que movilizara determinado peso. Si en ese primer intento no lograba llegar a su 1RM o ejecutaba más de una repetición, descansaba cinco minutos y lo volvía a intentar, con un peso mayor o menor, según fuera el caso. Se contabilizaba como $1 \mathrm{RM}$ al peso con el que era capaz de ejecutar solamente una repetición, realizando tanto la fase concéntrica como excéntrica del ejercicio, con la técnica correcta.

Posterior a ese primer día de medición, los sujetos participaron en tres sesiones experimentales, ordenadas de forman aleatoria, que consistieron en:

1. Ejercicio al $50 \%$ de $1 \mathrm{RM}$ (7 ejercicios, 2 series de 14 repeticiones, descanso de 45 segundos entre series).

2. Ejercicio al $70 \%$ de 1 RM (7 ejercicios, 2 series de 10 repeticiones, descanso de 45 segundos entre series).

3. Condición control (permanecer sentados durante $35 \mathrm{~min}$ ).

Para las sesiones de ejercicio se realizó un calentamiento de 10 minutos en banda sin fin, luego se realizaron los ejercicios CR a la intensidad que correspondía ese día, respetando el siguiente orden: jalón con polea abierto, extensión de rodilla con máquina, prensa de banca horizontal con barra, flexión de rodilla con máquina, flexión de codo en banco Scott con barra Z, extensión de codo polea con barra corta y prensa militar con máquina.

Al inicio de cada condición, se les solicitó a los participantes permanecer sentados durante cinco minutos para medir la PA tres veces, con un intervalo de tres minutos entre medición. Con dichos valores se obtuvo un promedio de la PA inicial (AE). Al finalizar cada condición experimental, se tomó de nuevo la PA inmediatamente terminado el tiempo de intervención (PE) y cada diez minutos hasta completar 60 minutos.

El protocolo de medición de esta variable consistió en medir la PA en el brazo izquierdo, el cual debía estar apoyado sobre una superficie plana a la altura del corazón. Durante la medición se le pidió al sujeto que mantuviera la espalda recostada en el respaldar de la silla, que guardara silencio y que evitara cruzar las piernas. 
Análisis estadístico. Para el análisis se utilizó el programa estadístico para las ciencias sociales (SPSS Inc. Chicago, Illinois, USA) versión 15.0; los valores de $p<0.05$ fueron aceptados como significativos. Para la estadística descriptiva se obtuvieron los promedios y desviaciones estándar de cada medición en cada condición, mientras que para la inferencial se utilizaron dos ANOVA de dos vías con medidas repetidas en ambos factores ( 3 condiciones $\times 8$ mediciones), uno para la PA sistólica (PAs) y otro para la diastólica (PAd). Además, se calcularon los efectos simples y post hoc de Tukey, en los casos donde se encontraron interacciones significativas.

\section{RESULTADOS}

En la Tabla 1 se muestran los promedios y desviaciones estándar de la PA tanto sistólica como diastólica en las tres condiciones durante las ocho mediciones.

Tabla 1

Estadística descriptiva de la presión arterial en cada condición y medición

\begin{tabular}{|c|c|c|c|}
\hline Condiciones & Mediciones & $\begin{array}{c}\text { Promedio } \pm \text { DS } \\
\text { PAs }(\mathrm{mmHg})\end{array}$ & $\begin{array}{l}\text { Promedio } \pm \text { DS } \\
\text { PAd }(\mathrm{mmH})\end{array}$ \\
\hline \multirow{8}{*}{$50 \%$ 1RM } & $\mathrm{AE}$ & $113.70 \pm 6.58$ & $70.90 \pm 8.46$ \\
\hline & PE & $124.30 \pm 14.34$ & $72.10 \pm 6.13$ \\
\hline & 10 & $112.70 \pm 8.23$ & $63.50 \pm 8.18$ \\
\hline & 20 & $113.40+8.63$ & $6240+6.91$ \\
\hline & 30 & $111.00 \pm 7.27$ & $62.80 \pm 6.19$ \\
\hline & 40 & $11090+724$ & $6480+713$ \\
\hline & 50 & $10990+872$ & $6580+6.86$ \\
\hline & 60 & $110.90 \pm 8.14$ & $65.30 \pm 7.40$ \\
\hline \multirow{8}{*}{$70 \%$ 1RM } & $\mathrm{AE}$ & $116.20 \pm 7.19$ & $70.60 \pm 6.58$ \\
\hline & PE & $125.00 \pm 14.07$ & $68.70 \pm 7.87$ \\
\hline & 10 & $112.00 \pm 5.18$ & $62.70 \pm 7.86$ \\
\hline & 20 & $107.90 \pm 7.09$ & $62.10 \pm 8.19$ \\
\hline & 30 & $106.10 \pm 6.34$ & $64.50 \pm 6.96$ \\
\hline & 40 & $109.20 \pm 6.23$ & $64.10 \pm 7.73$ \\
\hline & 50 & $106.90 \pm 4.86$ & $68.50 \pm 7.97$ \\
\hline & 60 & $107.60 \pm 4.19$ & $69.50 \pm 7.82$ \\
\hline \multirow{8}{*}{ Control } & $\mathrm{AE}$ & $118.00 \pm 8.84$ & $72.40 \pm 7.35$ \\
\hline & PE & $118.60 \pm 8.30$ & $69.70 \pm 8.11$ \\
\hline & 10 & $114.00 \pm 7.83$ & $69.80 \pm 9.11$ \\
\hline & 20 & $116.20 \pm 8.05$ & $73.10 \pm 7.96$ \\
\hline & 30 & $114.40 \pm 8.19$ & $72.70 \pm 7.51$ \\
\hline & 40 & $114.60 \pm 7.13$ & $73.20 \pm 8.46$ \\
\hline & 50 & $116.10 \pm 6.20$ & $74.00 \pm 6.18$ \\
\hline & 60 & $115.40 \pm 6.41$ & $75.40 \pm 5.12$ \\
\hline
\end{tabular}

Nota: 1RM: una repetición máxima, AE: antes del ejercicio, PE: posterior al ejercicio, DS: desviación estándar, PAs: presión arterial sistólica, PAd: presión arterial diastólica. Fuente: elaboración propia.

En la Tabla 2 se aprecian los resultados de los análisis de varianza de dos vías. Como se puede notar, se encontraron interacciones significativas entre condiciones y mediciones, tanto para la PA sistólica $(p=0.003)$ como para la PA diastólica $(p<0.001)$. 
Tabla 2

Resultados obtenidos del análisis de varianza (ANOVA)

\begin{tabular}{cccc}
\hline Presión Arterial & Fuente & $\boldsymbol{F}$ & $\boldsymbol{p}$ \\
\hline Presión arterial & Condiciones & 4.408 & $\mathbf{0 . 0 2 8}^{\boldsymbol{}}$ \\
sistólica & Mediciones & 92.16 & $\mathbf{0 . 0 0 3}^{\boldsymbol{*}}$ \\
& Condiciones x mediciones & 69.41 & $<\mathbf{0 . 0 0 1}^{\boldsymbol{}}$ \\
\hline Presión arterial & Condiciones & 0.249 & 0.782 \\
diastólica & Mediciones & 30.48 & $\mathbf{0 . 0 0 3}^{\boldsymbol{*}}$ \\
& Condiciones x mediciones & 16.92 & $\mathbf{0 . 0 0 1}^{\boldsymbol{*}}$ \\
\hline
\end{tabular}

Fuente: elaboración propia.

Debido a que las interacciones fueron significativas, se procedió a realizar los post hoc de efectos simples, tanto para la PAs como para la PAd. No se encontraron diferencias estadísticamente significativas al inicio de la sesión, lo cual indica que los sujetos comenzaron las tres condiciones en estado similar; tampoco se detectaron cambios significativos en las mediciones de la condición control, tanto para la PAs como para la PAd. En la Figura 1 se observa el comportamiento de la PAs según la condición experimental y el momento de medición.

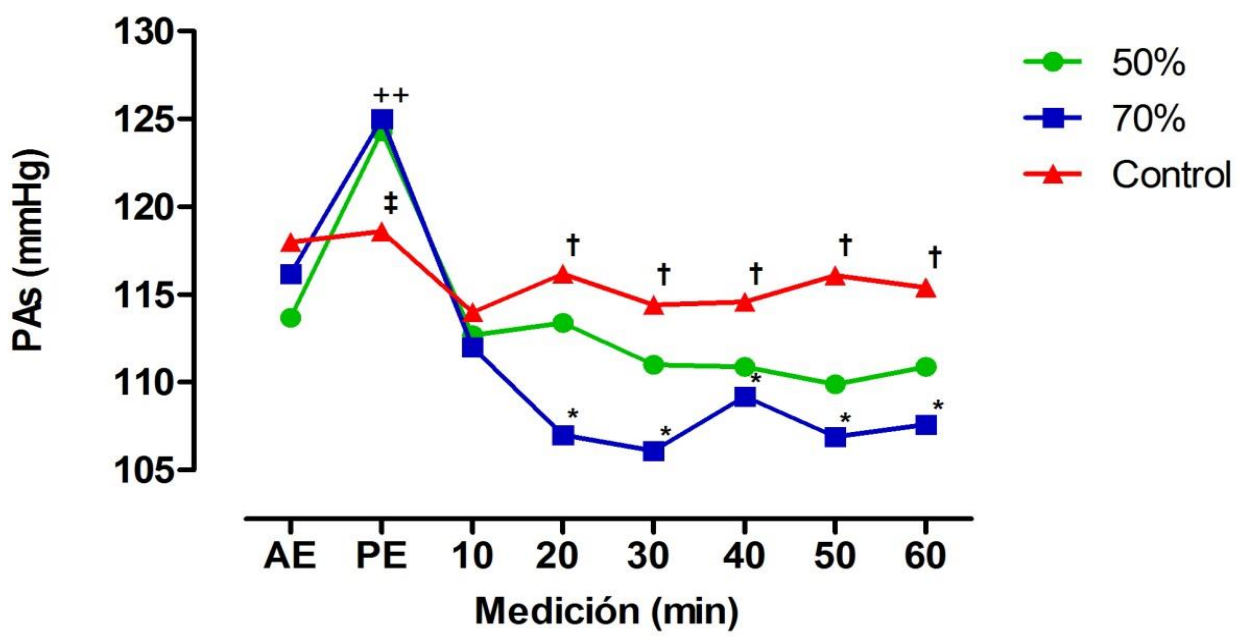

Figura 1. Presión arterial sistólica. Nota: PAs: presión arterial sistólica; AE: antes del ejercicio; PE: posterior al ejercicio. Símbolos que muestra diferencias significativas $(p<0.05)$ entre: ${ }^{++}$: la PAs de la medición PE y la PAs de las demás mediciones dentro de las condiciones de $50 \%$ y $70 \%$.‡: la PAs de la medición PE cuando se realizó ejercicio al $50 \%$ y $70 \%$ y la PAs de la condición control en ese momento. ${ }^{\dagger}$ : la PAs de la condición control y la PAs del $70 \%$ en ese momento de medición. ": la PAs de ese momento y la PAs medida AE. Fuente: elaboración propia.

Cuando se analiza lo sucedido dentro de cada condición experimental se encuentra que: la PAs aumenta significativamente luego de hacer ejercicio al $50 \%$ y $70 \%$ de intensidad, para luego decrecer en los minutos posteriores (símbolo ++), mientras que al 70\%, la PAs presenta valores significativamente menores a partir del minuto 20, en comparación con los valores previos al ejercicio (símbolo *).

$$
\text { - } 7 \text { - }
$$

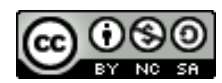


Al contrastar la PA de las condiciones de ejercicio con la condición control, se obtiene que la PAs es significativamente mayor cuando se realiza ejercicio en comparación a cuando no se realiza (símbolo $\ddagger$ ), y significativamente menor a partir del minuto 20 , si se realiza ejercicio al $70 \%$ de intensidad (símbolo †). En la Figura 2 se observa el comportamiento de la PAd según la condición experimental y el momento de medición.

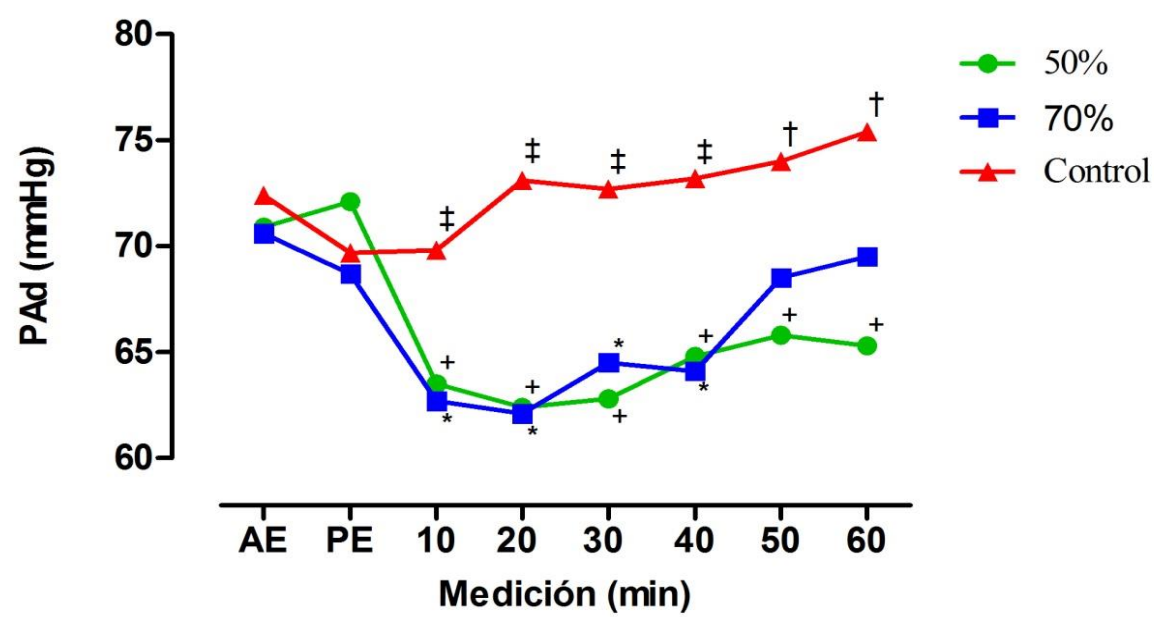

Figura 2. Presión arterial diastólica. Nota: Abreviaturas: PAd: presión arterial diastólica; AE: antes del ejercicio; PE: posterior al ejercicio. Símbolos que muestra diferencias significativas $(p<0.05)$ entre: +: la PAd de ese momento y la PAd de la medición AE en la condición de ejercicio al $50 \%$. ¥: la PAd de la condición control y la PAd de las condiciones de ejercicio al $50 \%$ y $70 \%$ en esa medición. †: la PAd de la condición control y la PAd de la condición de ejercicio al $50 \%$. ": la PAd de ese momento y la PAd medida AE en la condición de ejercicio al $70 \%$. Fuente: elaboración propia.

Cuando se analiza lo sucedido dentro de cada condición experimental, se encuentra que: en la condición de ejercicio al $50 \%$ de intensidad, la PAd presenta valores significativamente menores del minuto 10 al minuto 60, en comparación con el valor previo al ejercicio (símbolo +); mientras que al $70 \%$ de intensidad, la PAd presenta valores significativamente menores del minuto 10 al minuto 40, en comparación con el valor previo al ejercicio (símbolo *).

Cuando se confronta la PAd de las condiciones de ejercicio con la condición control, se obtiene que la PAd posterior al ejercicio de $50 \%$ es significativamente menor que la condición control del minuto 10 al minuto 60 (símbolo †), mientras que la PAd posterior al ejercicio de $70 \%$ es significativamente menor que la condición control desde el minuto 10 hasta el minuto 40 (símbolo $¥$ ).

\section{DISCUSIÓN}

Los principales resultados del presente estudio indican que el realizar ejercicio CR al $70 \%$ provoca durante los 60 min de medición una PAs menor a cuando no se realiza ejercicio; mientras que la PAd disminuye significativamente durante $60 \mathrm{~min}$ y $40 \mathrm{~min}$ al ejercitar al $50 \%$ y $70 \%$ de $1 \mathrm{RM}$, respectivamente. 
A pesar de que un metaanálisis reciente a nivel general mostró que, indistintamente de la intensidad a la que se realiza el ejercicio, esta produce efecto hipotensor (Carpio et al., 2016), dicha generalización se debe observar con cuidado si se desea obtener el mejor resultado al prescribir el ejercicio, ya que no toma en cuenta la edad de las personas participantes en los diferentes estudios, ni el tiempo de medición de la presión arterial, ni las diferencias metodológicas en las que se realizaron las mediciones de las repeticiones máximas.

El hecho de que los valores sistólicos y diastólicos iniciales de las tres condiciones no hayan mostrado diferencias significativas demuestra que los sujetos comenzaron con presiones arteriales similares durante los tres días en que participaron en los tratamientos. El mantenimiento de la presión arterial relativamente estable durante la condición control permite atribuir la respuesta de la PA al efecto agudo del ejercicio.

Aunque no se midieron las presiones arteriales durante el ejercicio, los valores obtenidos inmediatamente después de culminado el ejercicio CR son una aproximación a lo que pudo estar ocurriendo durante el mismo. El hecho de que la PAs estuviera levemente elevada (alrededor de $7 \mathrm{mmHg}$ ) luego de realizar ejercicio al $50 \%$ y al $70 \%$ de $1 \mathrm{RM}$, mientras que la diastólica mantuvo sus valores similares, reafirma lo expuesto por Carpio y Solera (2012), en el sentido de que tras el ejercicio CR, los valores de PAs de mujeres hipertensas medicadas de aproximadamente 53 años fueron significativamente mayores al realizar ejercicio aeróbico, CR y concurrente, en comparación con la condición control, mientras que la diastólica se mantuvo estable cinco minutos posterior a la finalización del ejercicio.

La PA está regulada por un complejo sistema de procesos que influyen no solo el gasto cardiaco sino también el volumen de eyección, la frecuencia cardiaca, las resistencias vasculares y la capacitancia venosa (Fiedler y Gaurzong, 2005). A nivel del efecto hipotensor del ejercicio, estudios recientes indican que la disminución de la resistencia vascular juega un papel muy importante, y esta parece deberse a la disminución del flujo simpático y al aumento de la liberación de sustancias vasodilatadoras, como el óxido nítrico, producto del ejercicio (Hamer, 2006).

En el estudio de Keese et al. (2011) se manipuló el tipo de ejercicio, mas no las intensidades del mismo, y se encontraron disminuciones tanto de la PAs como de la PAd posterior a cualquiera de las tres condiciones: ejercicio CR al $80 \%$ de $1 \mathrm{RM}$, aeróbico al $65 \%$ del consumo de oxígeno pico y concurrente (combinación de ambos ejercicios mencionados). No obstante, surge la duda de si las disminuciones de las presiones arteriales respondían a los tipos de ejercicio o a las intensidades a las que se ejercitaron.

Si se comparan los resultados del ejercicio CR después de entrenar al 50\% y 70\% con los resultados obtenidos por Gamboa y Solera (2014) luego de trabajar a las mismas intensidades, pero realizando ejercicio aeróbico, se nota que ambos tipos de ejercicio son beneficiosos para lograr la reducción de la PA. De esta forma, mientras el ejercicio aeróbico realizado al $50 \%$ y $70 \%$ disminuye la PAs y no produce cambio en la PAd, el ejercicio CR disminuye la PAs solamente si se realiza al $70 \%$, pero reduce la PAd luego de realizar ejercicio al $50 \%$ y $70 \%$ de $1 \mathrm{RM}$. Esto parece indicar que el ejercicio concurrente podría producir disminuciones de presiones arteriales sistólicas y diastólicas al trabajarse a un $60 \%$ de intensidad, lo cual podría analizarse en un futuro estudio. 
Fisiológicamente se conoce que, durante el ejercicio aeróbico, la PAs se eleva producto del aumento en la frecuencia cardiaca y, por ende, el gasto cardiaco, mientras la PAd se mantiene relativamente constante (Williams et al., 2007; Gamboa y Solera, 2014). En contraparte, durante el ejercicio $C R$, al haber una mayor resistencia periférica, la PAs también se ve incrementada, pero en menor medida que cuando se realiza ejercicio aeróbico. Asimismo, la PAd también se eleva; no obstante, esta elevación sucede momentáneamente durante cada repetición (Baum, Rüther, \& Essfeld, 2003). Por lo tanto, pareciera ser que la elevación de la PAd durante el ejercicio CR podría estimular la aparición del efecto hipotensor que no se reflejó durante el ejercicio aeróbico realizado en el estudio de Gamboa y Solera (2014).

La disminución significativa de la PAs posterior al ejercicio CR ejecutado al $70 \%$ pero no del $50 \%$ de $1 \mathrm{RM}$ contrasta con los resultados obtenidos por Rezk et al. (2006), quienes encontraron disminución de la PAs luego de realizar ejercicio CR al $40 \%$. La falta de efecto hipotensor en la PAs posterior a la intensidad de un $50 \%$ podría deberse al hecho de que nuestros participantes fueron en promedio 15 años mayores a los sujetos medidos por Rezk et al. (2006); y como se ha mencionado en otros estudios, con el paso de los años aumenta la rigidez arterial producto de la destrucción progresiva de las fibras elásticas, de la disminución de la densidad capilar y del aumento del grueso de la pared de las arteriolas.

El problema de estos cambios estructurales es que, a su vez, resultan en cambios funcionales que aumentan la resistencia vascular y limitan la respuesta a los agentes vasodilatadores que se liberan durante el ejercicio (Canuto et al., 2011). En este aspecto, en un estudio futuro se podría comparar el efecto de diferentes intensidades de ejercicio sobre la presión arterial de personas con diferentes edades, para determinar si el efecto hipotensor del ejercicio varía según la edad de la persona.

Una limitación de este estudio consistió en que se midió la presión arterial en reposo durante solamente $60 \mathrm{~min}$, por lo que no se logró determinar la duración exacta del efecto hipotensor del ejercicio. Para futuros estudios se sugiere utilizar la medición ambulatoria, ya que permite registrar la presión arterial por 24 horas mientras la persona realiza sus actividades diarias y, a su vez, desenmascarar el síndrome de la gabacha blanca, el cual consiste en una elevación transitoria de la PA que les ocurre a ciertas personas cuando se les mide en un consultorio médico (Pickering, Phil, Shimbo, \& Hass, 2006).

En conclusión, se demuestra que, indistintamente de la intensidad a la que las personas normotensas realicen el ejercicio CR, se produce un efecto hipotensor en la hora posterior a su finalización.

\section{REFERENCIAS}

Baum, K., Rüther, T., \& Essfeld, D. (2003). Reduction of blood pressure response during strength training through intermittent muscle relaxations. International Journal of Sports Medicine, 24(6), 441-445. Doi: https://doi.org/10.1055/s-2003-41172

Bermudes, A.M., Vassallo, D.V., Vasquez, E.C., \& Lima, E.G. (2003). Ambulatory blood pressure monitoring in normotensive individuals undergoing two single exercise sessions. Resistive exercise training and aerobic exercise training. Arq Bras -10 - 
Cardiol, 82(1), 65-71. Recuperado de: http://www.scielo.br/pdf/abc/v82n1/ en a06v82n1.pdf

Canuto, P.M.B.C., Nogueira, I.D.B., Cunha, E.S., Ferreira, G.M.H., Mendoça, K.M.P.P., Costa, F.A., \& Nogueira, P.A.M.S. (2011). Influence of resistance training performed at different intensities and same work volume over BP of elderly hypertensive female patients. Revista Brasileira de Medicina do Esporte, 17(4), 246-249. Doi: https://doi.org/10.1590/S1517-86922011000400006

Cardoso, C. G., Gomides, R.S., Queiroz, A.C.C., Pinto, L.G., Lobo, F.S., Tinucci, T.,... Forjaz, C.L.M. (2010). Acute and chronic effects of aerobic and resistance exercise on ambulatory blood pressure. Clinics, 65(3), 317-25. Doi: https://doi.org/10.1590/S1807-59322010000300013

Carpio, E. y Solera, A. (2012). Efecto agudo de circuitos de ejercicios aeróbicos, contra resistencia o combinados sobre la presión arterial de mujeres con hipertensión. Pensar en Movimiento: Revista de Ciencias del Ejercicio y la Salud, 10(2), 1-12. Doi: https://doi.org/10.15517/pensarmov.v10i2.3454

Carpio-Rivera, E., Moncada-Jiménez, J., Salazar-Rojas, W., \& Solera-Herrera, A. (2016). Acute effects of exercise on blood pressure: A meta-analytic investigation. Arquivos Brasileiros de Cardiologia. 106(5), 422-33. Doi: https://doi.org/10.5935/abc.20160064

Chobanian, A., Bakris, H.R, Black, H.R., Cuschman, W.C., Green, L.A., Izzo, J.L.,... Rocella, E.J. (2003). The $7^{\text {th }}$ report of the Joint Nacional Committee on Detection, Evaluation and Treatment of High Blood Pressure. (JNC VII). JAMA, 289(19), 2560-2572. Doi: https://doi.org/10.1001/jama.289.19.2560

Del Valle, M., Manonelles, P., de Teresa, C., Franco, L., Luengo, E. y Gaztañaga, T. (2015). Prescripción de ejercicio físico en la prevención y tratamiento de la hipertensión arterial. Documento de Consenso de la Sociedad Española de Medicina del Deporte (SEMED-FEMEDE). Arch Med Deporte, 32(5), 281-312. Recuperado de: http://femede.es/documentos/Consenso prescripcion ejercicio HTA.pdf

Fiedler, E. y Gaurzong, C. (2005). Epidemiología y patogenia de la hipertensión arterial esencial, rol de angiotensina II, óxido nítrico y endotelina. Acta Médica Costarricense; 47(3), 109-17. Recuperado de http://www.actamedica. medicos.cr/index.php/Acta Medica/article/view/190/172

Fuchs, J.R. (2005). Hipertensión arterial. Acta Médica Costarricense; 47(3),. Recuperado de http://www.actamedica.medicos.cr/index.php/Acta Medica/ article/view/188

Gamboa, M. y Solera, A. (2014). Efecto agudo de dos intensidades de ejercicio aeróbico sobre la presión arterial en reposo de personas normotensas. Revista Andaluza de Medicina del Deporte, 7(3), 101-105. Doi: https://doi.org/10.1016/S18887546(14)70070-X 
Hamer, M. (2006). The anti-hypertensive effects of exercise: integrating acute and chronic mechanisms. Sports Medicine, 36(2), 109-116. Doi: https://doi.org/10.2165/00007256-200636020-00002

James, P.A., Oparil, S., Carter, B.L., Cushman, W.C., Dennison-Himmelfarb, C., Handler, J.,...Ortiz, E. (2014). Evidence-based guideline for the management of high blood pressure in adults. Report from the panel members appointed to the eighth Joint National Committee (JNC 8). JAMA, 311(5), 507-520. Doi:10.1001/jama.2013.284427 https://doi.org/10.1001/jama.2013.284427

Keese, F., Farinatti, P., Pescatello, L., \& Monteiro, W. (2011). A comparison of the immediate effects of resistance, aerobic and concurrent exercise on postexercise hypotension. Journal of Strength \& Conditioning Research, 25(5), 1429-1436. Doi: https://doi.org/10.1519/JSC.0b013e3181d6d968

Kenney, M.J., \& Seals, D.R. (1993). Postexercise Hypotension. Key features, mechanisms, and clinical significance. Hypertension, 22(5), 653-64. Doi: https://doi.org/10.1161/01.HYP.22.5.653

Liu, S., Goodman, J., Nolan, R., Lacombe, S., \& Thomas, S.H. (2012). Blood pressure response to acute and chronic exercise are related in prehypertension. Medicine \& Science Sports \& Exercise, 44(2), 1644-1652. Doi: https://doi.org/10.1249/MSS.0b013e31825408fb

Pickering, T.G., Phil, D., Shimbo, D., \& Hass, D. (2006). Ambulatory blood-pressure monitoring. The New England Journal of Medicine, 354, 2368-2374. Doi: https://doi.org/10.1056/NEJMra060433

Rezk, C.C., Marrache, R.C.B., Tinucci, T., Mion, D., \& Forjaz, C.L.M. (2006). Postresistance exercise hypotension, hemodynamics, and heart rate variability: influence of exercise intensity. European Journal of Applied Physiology, 98 (1), 105-112. Doi: https://doi.org/10.1007/s00421-006-0257-y

Williams, M.A., Haskell, W.L., Ades, P.A., Amsterdam, E.A., Bittner, V., Franklin, B.A.,...Stewart, K.J. (2007). Resistance Exercise in Individuals with and without cardiovascular diseases: 2007 Update. A scientific statement from de American Heart Association Council on Clinical Cardiology and Council on Nutrition, Physical Activity and Metabolism. Circulation, 116, 572-584. Doi: https://doi.org/10.1161/CIRCULATIONAHA.107.185214

Participación: A- Financiamiento, B- Diseño del estudio, C- Recolección de datos, D- Análisis estadístico e interpretación de resultados, E- Preparación del manuscrito. 